\title{
Study of Thermal Decomposition of the Copolymer Based on Polyethylene Glycol Fumarate with Acrylic Acid
}

\author{
Meyram Burkeyev, Yerkeblan Tazhbayev, Abylaikhan Bolatbay (D), Yelena Minayeva, \\ Santay Davrenbekov, Yermauyt Nassikhatuly, Anna Kovaleva, Kamshat Kutzhanova, \\ and Akerke Kazhmuratova
}

Chemical Faculty, Karaganda Buketov University, Karaganda 100000, Kazakhstan

Correspondence should be addressed to Abylaikhan Bolatbay; abylai_bolatbai@mail.ru

Received 13 January 2022; Accepted 8 February 2022; Published 23 February 2022

Academic Editor: Marinos Pitsikalis

Copyright (c) 2022 Meyram Burkeyev et al. This is an open access article distributed under the Creative Commons Attribution License, which permits unrestricted use, distribution, and reproduction in any medium, provided the original work is properly cited.

\begin{abstract}
Thermal decomposition of the copolymer based on polyethylene glycol fumarate with acrylic acid (p-EGF:AA) at four different heating rates in a nitrogen atmosphere has been investigated by the dynamic thermogravimetry (TGA) method. The TGA curves show that the decomposition process occurs in several stages. The experimental data processed by the Kissinger-Akahira-Sunose and Friedman isoconversion methods made it possible to calculate the activation energy of the main decomposition stage. According to the calculations results, one can see that the values found by these methods do not strongly depend on the conversion. The Achar-Brindley-Sharp method has been applied to determine the compensation parameters. Then, using the compensation effect, the invariant kinetic parameters of the decomposition processes have been found. An experimental curve has been plotted to find the reaction model in comparison with theoretical ones.
\end{abstract}

\section{Introduction}

Thermal analysis is one of the most rapidly developing methods for studying physicochemical and chemical transformations in the substance. According to the International Confederation for Thermal Analysis and Calorimetry (ICTAC), thermal analysis methods include a group of measurement methods in which the dependence of the mass, heat flux, and heat capacity of the test substance on temperature under programmed heating conditions is established. Thermal analysis methods have found the most widespread use in scientific research, and interest in them is steadily growing, due to the speed of obtaining information [1-3].

Thermal analysis is also widely used in the study of high molecular weight compounds and is an extremely valuable tool in the study of their thermal stability [4-6]. Unsaturated polyester resins are among poorly studied ones and have practical interest as objects of research. Solutions of unsaturated polyester resins in monomers have an increased viscosity, and the presence of unsaturated maleate or fumarate groups makes them capable of entering into copolymerization reactions with vinyl monomers. For the first time, the interaction reactions of unsaturated polyester resins with vinyl monomers were called copolymerization reactions by Boenig [7]. At the same time, prior to our studies [8-10], there was no information on the copolymerization of unsaturated polyester resins with hydrophilic monomers, which were tested as moisture sorbents and matrix-bases for creating metal-polymer complexes.

The literature contains data on the study of the thermal stability of copolymers of unsaturated polyester resins with hydrophobic monomers in a wide temperature range [11]. The results of thermogravimetric analysis have shown that these copolymers are thermally stable up to $250-300^{\circ} \mathrm{C}$, after which thermal degradation occurs, which proceeds in several stages [12].

We have previously investigated the thermal decomposition of some copolymers of unsaturated polyester resins 
with ionic monomers, in which the influence of copolymers composition on their thermal stability has been shown. The basic kinetic parameters of thermal degradation of copolymers have been found using integral and differential methods [13-15]. Although, as noted in [16], there is no unified approach to determine the kinetic triplet, the use of different methods and their comparison allows to obtain a mathematical explanation describing the complex reactions during thermal decomposition of solids.

In this work, for the first time, studies on the thermal degradation of copolymers of polyethylene glycol fumarate with acrylic acid have been carried out in an inert medium at different heating rates with a description of the reaction model.

\section{Experimental Part}

2.1. Synthesis of Copolymer. Polyethylene glycol fumarate (p-EGF) was obtained by the polycondensation reaction of fumaric acid with ethylene glycol in a stream of nitrogen with heating and stirring [17]. Copolymerization of unsaturated polyester resin with acrylic acid was carried out in the presence of a radical copolymerization initiator, namely, benzoyl peroxide. Copolymers were obtained in bulk at a temperature of $293 \mathrm{~K}$ [18]. The synthesized copolymers were washed with dioxane to purify them from the residues of the unreacted monomer mixture with further filtration, and then, the polymers were dried in a vacuum oven at a temperature of $313 \mathrm{~K}$ until a constant weight was established.

2.2. Thermal Analysis. Investigation of thermal properties of the p-EGF:AA copolymer was carried out on a device for synchronous thermal analysis Labsys Evolution TG/DTA from Setaram in a dynamic mode in the temperature range of $30-600^{\circ} \mathrm{C}$. The samples were heated in an $\mathrm{Al}_{2} \mathrm{O}_{3}$ crucible at a rate of $2.5,5,10$, and $20^{\circ} \mathrm{C} / \mathrm{min}$ in a nitrogen atmosphere at a flow rate of $30 \mathrm{~mL} / \mathrm{min}$. The instrument for thermogravimetric studies and heat flux were calibrated using $\mathrm{CaCO}_{3}$ and in standards, respectively.

2.3. Kinetic Analysis. The thermal decomposition kinetics is usually expressed by the following equation:

$$
\frac{\mathrm{d} a}{\mathrm{~d} t}=k(T) f(a),
$$

where $k$ is a constant of decomposition rate, $t$ is the reaction time, and $\alpha$ is the conversion rate, which is described as

$$
\alpha=\frac{m_{i}-m_{t}}{m_{i}-m_{f}},
$$

where $m_{i}, m_{t}$, and $m_{f}$ are the masses at the beginning, time $t$, and the reaction end.

The decomposition rate constant $(k(T))$ is a function of temperature and is usually described by the Arrhenius equation. Hence, equation (1) can be written as follows:

$$
\frac{\mathrm{d} a}{\mathrm{~d} t}=A \exp \left(\frac{-E a}{R T}\right) f(a),
$$

where $A$ is a preexponential factor, $E a$ is an activation energy, $R$ is the universal gas constant $\left(8.314 \mathrm{Jmol}^{-1} \mathrm{~K}^{-1}\right)$, and $T$ is a temperature $(\mathrm{K})$.

Integral and differential methods were used to obtain the activation energy $(E \alpha)$.

2.3.1. The Kissinger-Akahira-Sunose Method. The studies by Kissinger and Akahira and Sunose $[19,20]$ are based on the following expression:

$$
\ln \left(\frac{\beta_{i}}{T_{\alpha, i}^{2}}\right)=\text { Const }-\left(\frac{E a}{R T \alpha}\right) .
$$

The activation energy can be obtained from the dependence graph $\ln \left(\beta_{i} / T_{\alpha, i}^{2}\right)$ from $1 / T_{\alpha}$, for given value of the conversion degree $\alpha$, where the slope is equal to $E a / R$.

2.3.2. The Friedman Method. The Friedman method is the most common differential isoconversion method [21]. The method is based on the following equation:

$$
\ln \left[\beta_{i}\left(\frac{\mathrm{d} \alpha}{\mathrm{d} T}\right)_{\alpha, i}\right]=\ln \left[f(\alpha) A_{\alpha}\right] \frac{E_{a}}{R T_{\alpha, i}} .
$$

For each given $\alpha$, the value of $\mathrm{E}_{a}$ is determined by the slope of the graph $\ln \left[\beta_{i}(\mathrm{~d} \alpha / \mathrm{d} T)_{\alpha, i}\right]$ from $1 / T_{\alpha, i}$. Index $i$ is introduced to indicate the different temperature programs. $T_{\alpha, i}$ is the temperature at which the conversion degree $\alpha$ is achieved in the $i^{\text {th }}$ temperature program.

2.3.3. Model Fitting Method and IKP Method. The method of invariant kinetic parameters and the model fitting method were used in this work to determine the reaction model and preexponential factor. The Achar-Brindley-Sharp differential method [22] is obtained by taking the logarithm of equation (1):

$$
\ln \left(\frac{\mathrm{d} a / \mathrm{d} t}{f(a)}\right)=\ln A-\frac{E}{R T} .
$$

The left side of the equation (6) for a given analytical form $f(\alpha)$ should be a straight line, the slope and intersection of which allow one to estimate the activation energy and preexponential factor, respectively. The models used in this study are given in Table 1; although the parameters vary over a wide range of $f(\alpha)$, they all exhibit a strong correlation known as the compensation effect:

$$
\ln A_{i}=a^{*} E_{i}+b^{*} .
$$

Using the ratio of the apparent compensation effect, compensation parameters are determined for each heating rate. Thus, the graph $a^{*}$ vs. $b^{*}$ is a straight line, the parameters of which make it possible to estimate the invariant kinetic parameters [23]:

$$
b^{*}=\ln A+a^{*} E .
$$

After both parameters are determined, the reaction model can be numerically reconstructed in any integral or 
TABLE 1: Some of the kinetic models used in the solid-state kinetics.

\begin{tabular}{lcccc}
\hline & Reaction model & Code & $f(\alpha)$ & $g(\alpha)$ \\
\hline 1 & Power law & P4 & $4 \alpha^{3 / 4}$ & $\alpha^{1 / 4}$ \\
2 & Power law & P3 & $3 \alpha^{2 / 3}$ & $\alpha^{1 / 3}$ \\
3 & Power law & P2 & $2 \alpha^{1 / 2}$ & $\alpha^{1 / 2}$ \\
4 & Power law & P2/3 & $2 / 3 \alpha^{-1 / 2}$ & $\alpha^{3 / 2}$ \\
5 & D1 & $1 / 2 \alpha^{-1}$ & $-\ln (1-\alpha)$ \\
6 & One-dimensional diffusion & F1 & $1-\alpha$ & {$[-\ln (1-\alpha)]^{1 / 4}$} \\
7 & Mampel (first order) & A4 & $4(1-\alpha)[-\ln (1-\alpha)]^{3 / 4}$ & {$[-\ln (1-\alpha)]^{1 / 3}$} \\
8 & Avrami-Erofeev & A3 & $3(1-\alpha)[-\ln (1-\alpha)]^{2 / 3}$ & {$[-\ln (1-\alpha)]^{1 / 2}$} \\
9 & Avrami-Erofeev & A2 & $2(1-\alpha)[-\ln (1-\alpha)]^{1 / 2}$ & $\left.[1-(1-\alpha)]^{1 / 3}\right]^{2}$ \\
10 & Avrami-Erofeev & D3 & $\left.3 / 2(1-\alpha)^{2 / 3}[1-(1-\alpha)]^{1 / 3}\right]^{-1}$ & $1-(1-\alpha)^{1 / 3}$ \\
11 & Three-dimensional diffusion & R3 & $3(1-\alpha)$ & $1-(1-\alpha)^{1 / 2}$ \\
12 & Contracting sphere & R2 & $2(1-\alpha)$ & $(1-\alpha) \ln (1-\alpha)+\alpha$ \\
13 & Contracting cylinder & D2 & {$[-\ln (1-\alpha)]^{-1}$} & \\
\hline
\end{tabular}

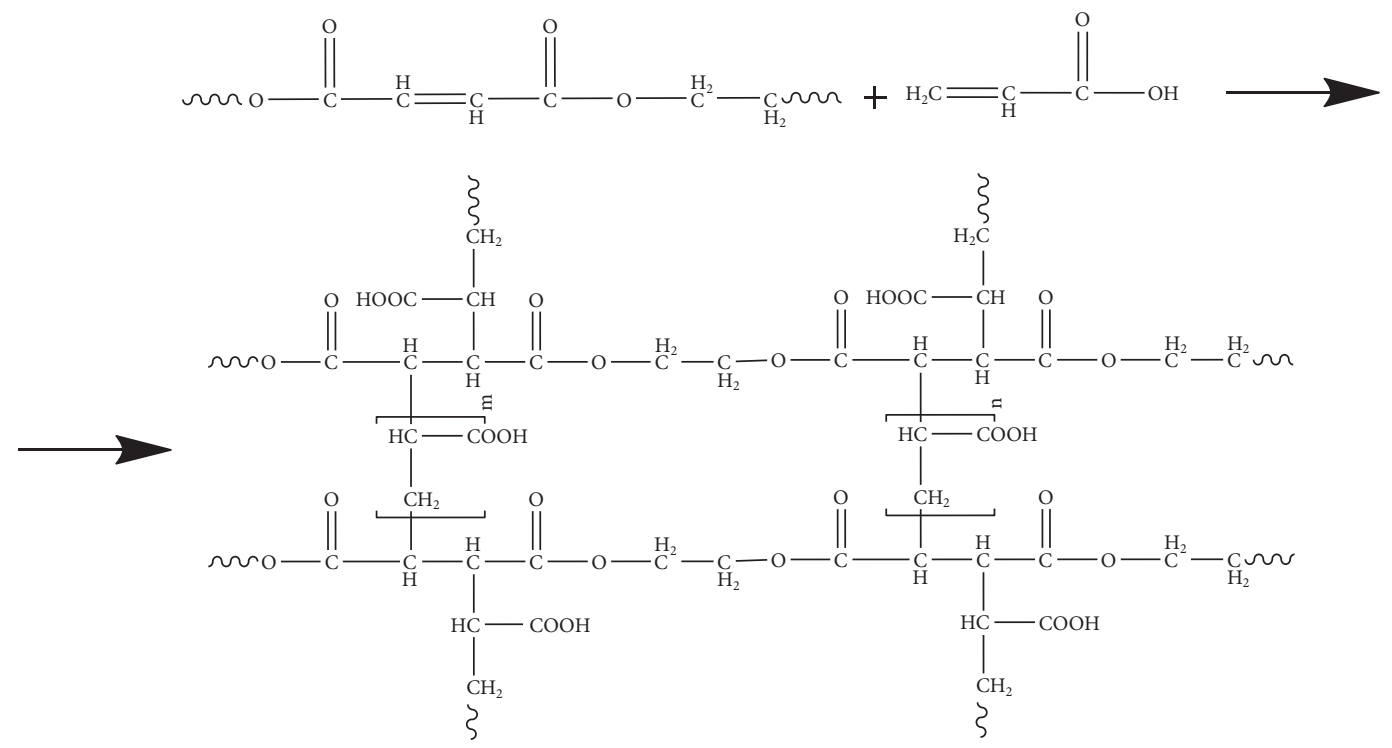

Scheme 1: Brief scheme of copolymerization of p-EGF with AA.

differential form. The integral form can be restored by substituting the values of $E a$ and $A$ in the equation [24]:

$$
g(\alpha)=\frac{A}{\beta} \int_{0}^{T_{\alpha}} \exp \left(\frac{-E}{R T}\right) \mathrm{d} T .
$$

\section{Results and Discussion}

3.1. Thermogravimetric Analysis. Previously, we synthesized copolymers with high moisture-absorbing properties at various compositions of the initial mixture by the radical copolymerization reaction of unsaturated polyester resins [18]. It has been shown that copolymerization proceeds according to the following scheme (Scheme 1).

The object of the study in this work is the p-EGF:AA copolymer of the composition 44.05:55.95 mol\%. Thermogravimetric analysis was carried out under dynamic conditions at four different heating rates. Figure 1(a) shows the dependence of the sample mass on temperature at all analyzed heating rates, and Figure 1(b) shows the curves of the decomposition rate of the copolymer.
Figure 1 shows that at the initial stage, the sample masses undergo insignificant changes associated with the release of moisture in the copolymer. The decomposition process begins at a temperature of $150^{\circ} \mathrm{C}$, followed by a gradual decrease in the mass of the samples with the release of volatile substances. The third stage of decomposition begins at a temperature of $261^{\circ} \mathrm{C}$ and ends at $434^{\circ} \mathrm{C}$, at the lowest heating rate (Figure $1(\mathrm{a})$ ). When the heating rate was $20^{\circ} \mathrm{C} / \mathrm{min}$, the initial and final decomposition temperatures shifted to 296 and $464^{\circ} \mathrm{C}$, respectively. The same tendency is observed on the DTG curve (Figure 1(b)). The DTG curve has a maximum decomposition rate and a shift to a higher temperature range at the maximum heating rate. The weight of the residue at the end was approximately $19-20 \%$ of the total weight of the sample. Table 2 provides the temperatures of the beginning, peak, and end of the third stage of decomposition.

3.2. Kinetic Analysis. The data from Table 2 have been used to plot dependencies of the conversion degree on the temperature, as shown in Figure 2. 


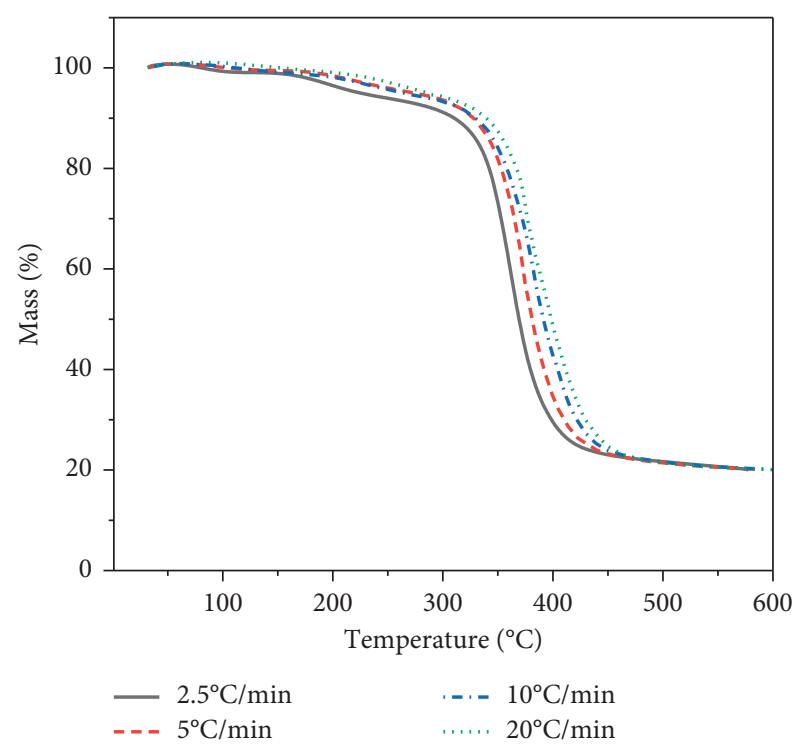

(a)

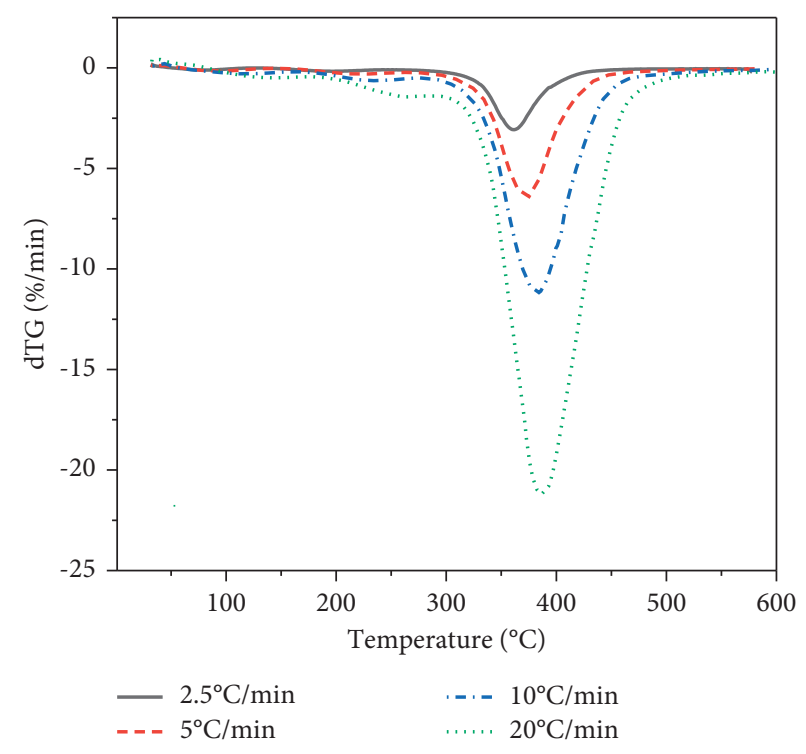

(b)

Figure 1: TGA (a) and DTG (b) curves of p-EGF:AA, $44.05: 55.95 \mathrm{~mol} \%$ obtained at different heating rates.

TABLE 2: Thermogravimetric data for p-EGF:AA copolymer, 44.05 : $55.95 \mathrm{~mol} \%$ at various heating rates.

\begin{tabular}{lccc}
\hline$\beta\left({ }^{\circ} \mathrm{C} / \mathrm{min}\right)$ & $\mathrm{T}_{\text {initial }}\left({ }^{\circ} \mathrm{C}\right)$ & $\mathrm{T}_{\text {peak }}\left({ }^{\circ} \mathrm{C}\right)$ & $\mathrm{T}_{\text {final }}\left({ }^{\circ} \mathrm{C}\right)$ \\
\hline 2.5 & 261 & 361 & 434 \\
5 & 275 & 372 & 452 \\
10 & 279 & 383 & 460 \\
20 & 296 & 387 & 464 \\
\hline
\end{tabular}

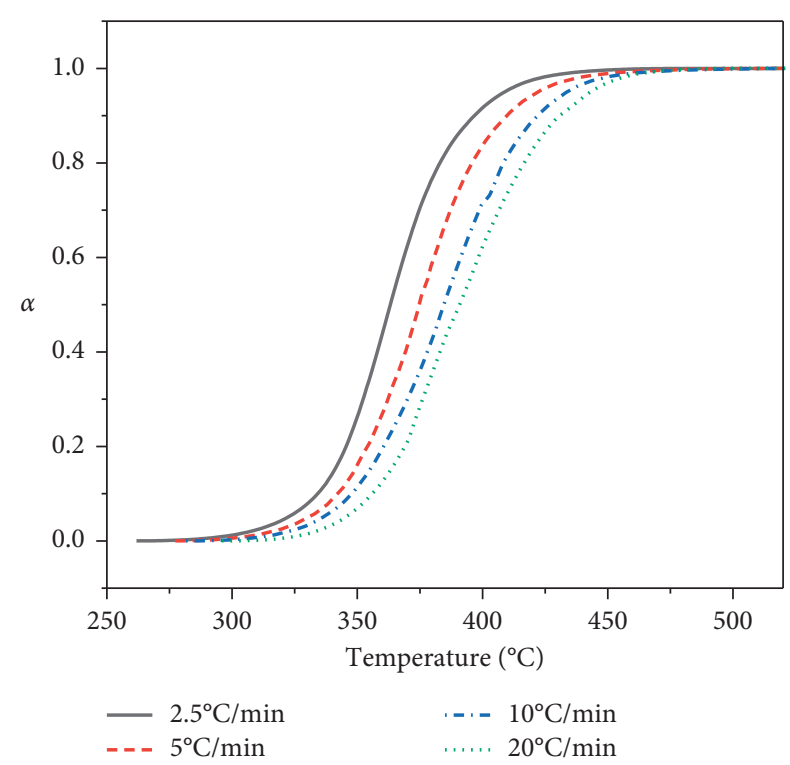

FIGURE 2: Dependence of the conversion degree $(\alpha)$ of the p-EGF: AA copolymer on temperature at different heating rates $(2.5,5,10$, and $20^{\circ} \mathrm{C} / \mathrm{min}$ ).

As shown in Figure 2, the curves shapes of the conversion degrees for all heating rates have a sigmoidal shape. The decomposition reaction with increasing temperature gradually accelerates and becomes more intense, followed by a slowdown. Also, it is possible to observe a shift of the curves by $10-15^{\circ} \mathrm{C}$ to the high temperatures region with an increase in the heating rate. The parameters dependences of the Kissinger-Akahira-Sunose and Friedman equations at various conversion degrees shown in Figures 3(a) and 3(b) were used to determine the kinetic parameters using the isoconversional principle.

As can be seen from the graphs, the points obtained by the Friedman differential method have a greater scatter (Figure 3(b)) than in the Kissinger-Akahira-Sunose (KAS) method, and as a consequence, the latter are more parallel to each other. The choice of these methods is due to the possibility of comparing the activation energy obtained by integral and differential methods, which allow assessing the validity of the assumptions made when deriving these equations. Figure 4 shows a graphical dependence of the activation energy on the conversion degree.

At the initial stage of decomposition, the activation energies found by two methods slightly increase and then tend to decrease. At a value of $\alpha>0.3$, the activation energy begins to decrease sharply and reaches a minimum at $\alpha=0.75$, with a subsequent increase until the completion of the thermal decomposition reaction. The dependence of the conversion degree on the activation energy is most informatively given in Table 3 .

Table 3 provides that degrees of correlation for both methods have the highest values at the beginning of the thermal decomposition reaction. Values with low correlation values are observed at conversions of $\alpha=0.3-0.45$. Thus, the Kissinger-Akahira-Sunose and Friedman methods made it possible to estimate the activation energy throughout the entire process without the preliminary use of reaction models.

In continuation of the work, the Achar-Brindley-Sharp method and the method of invariant kinetic parameters were 


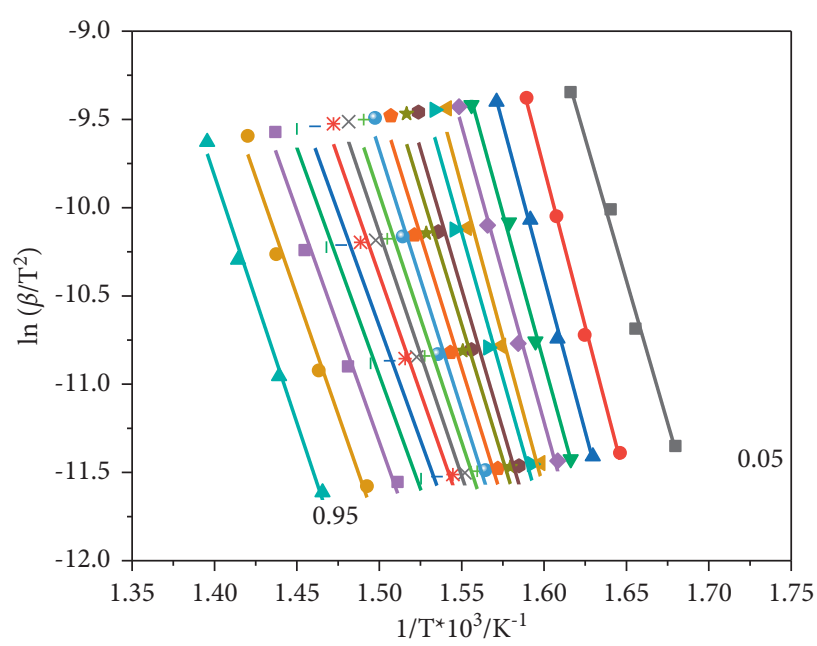

(a)

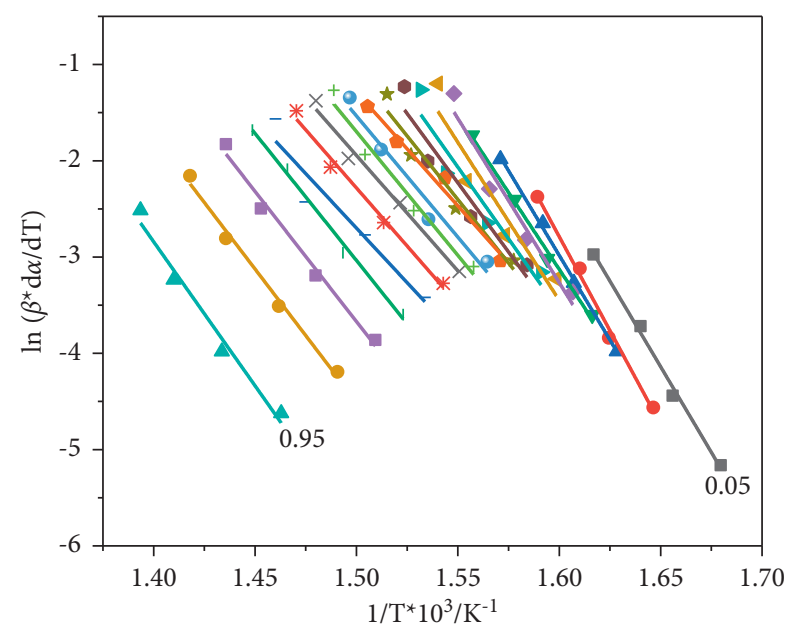

(b)

FIGURE 3: Graphical dependences of the Kissinger-Akahira-Sunose (a) and Friedman (b) equations for the p-EGF:AA copolymer at heating rates of $2.5,5,10$, and $20^{\circ} \mathrm{C} / \mathrm{min}$.

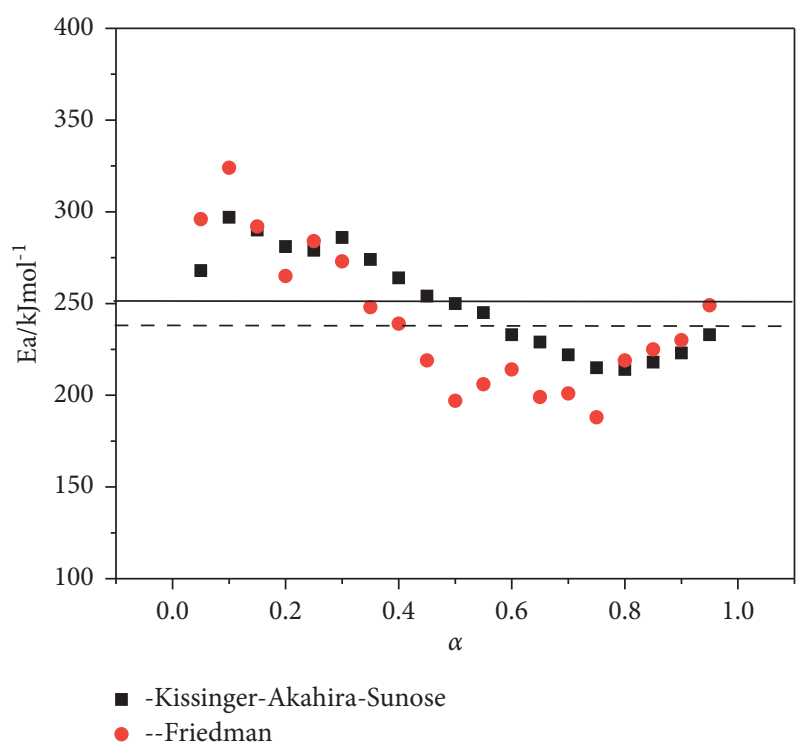

FIGURE 4: Dependence of the activation energy $\left(E_{a}\right)$ on the conversion degree $(\alpha)$ for the p-EGF:AA copolymer.

applied in order to determine the preexponential factor and the reaction model. It was stated in [24] that the ABS method was more accurate for calculating the kinetic parameters required to estimate the compensation parameters. Using equation (6), thirteen values of $E a$ and $\ln A$ (Figure 5) corresponding to the reaction models from Table 1 were obtained.

Figure 5 shows that the straight lines for all four heating rates lie very close and have a good correlation. The compensation parameters obtained by the Achar-BrindleySharp method are given in Table 4 .

Figure 6 shows a graph of $a^{*}$ vs. $b^{*}$ dependence.
The invariant kinetic parameters of the copolymer decomposition were found from the slope and intersection of the graph, namely, $E a=247 \mathrm{~kJ} / \mathrm{mol}$ and $\ln A=39$. The activation energy is in good agreement with the values obtained using isoconversion methods. Equation (9) was used to obtain a set of numerical values $g(\alpha)$ for various conversion rates. The dependence graph of the reaction model on the conversion degree has a profile reminiscent of an accelerating reaction (F1, D1, and R3). Therefore, the calculated data were compared to the previously selected theoretical graphs $g(\alpha)$.

As shown in Figure 7, a good conformity was found for the D3 model. 
TABLE 3: Activation energies according to Kissinger-Akahira-Sunose and Friedman.

\begin{tabular}{|c|c|c|c|c|}
\hline \multirow{2}{*}{$\alpha$} & \multicolumn{2}{|c|}{ Kissinger-Akahira-Sunose } & \multicolumn{2}{|c|}{ Friedman } \\
\hline & $E, \mathrm{~kJ} / \mathrm{mol}$ & $R^{2}$ & $E, \mathrm{~kJ} / \mathrm{mol}$ & $R^{2}$ \\
\hline 0.5 & $268 \pm 17$ & 0.9913 & $296 \pm 15$ & 0.9948 \\
\hline 0.1 & $297 \pm 20$ & 0.9976 & $324 \pm 17$ & 0.9944 \\
\hline 0.15 & $290 \pm 10$ & 0.9976 & $292 \pm 9$ & 0.9982 \\
\hline 0.2 & $281 \pm 13$ & 0.9956 & $265 \pm 13$ & 0.9952 \\
\hline 0.25 & $279 \pm 16$ & 0.9934 & $284 \pm 45$ & 0.9512 \\
\hline 0.3 & $286 \pm 29$ & 0.9796 & $273 \pm 63$ & 0.9032 \\
\hline 0.35 & $274 \pm 33$ & 0.9720 & $248 \pm 53$ & 0.9158 \\
\hline 0.4 & $264 \pm 36$ & 0.9642 & $239 \pm 48$ & 0.9242 \\
\hline 0.45 & $254 \pm 34$ & 0.9660 & $219 \pm 34$ & 0.9546 \\
\hline 0.5 & $250 \pm 27$ & 0.9764 & $197 \pm 19$ & 0.9808 \\
\hline 0.55 & $245 \pm 22$ & 0.9838 & $209 \pm 28$ & 0.9660 \\
\hline 0.6 & $233 \pm 30$ & 0.9687 & $214 \pm 26$ & 0.9714 \\
\hline 0.65 & $229 \pm 21$ & 0.9838 & $199 \pm 17$ & 0.9853 \\
\hline 0.7 & $222 \pm 20$ & 0.9841 & $201 \pm 15$ & 0.9890 \\
\hline 0.75 & $215 \pm 20$ & 0.9825 & $188 \pm 40$ & 0.9123 \\
\hline 0.8 & $214 \pm 18$ & 0.9860 & $219 \pm 12$ & 0.9940 \\
\hline 0.85 & $218 \pm 18$ & 0.9863 & $225 \pm 18$ & 0.9878 \\
\hline 0.9 & $223 \pm 18$ & 0.9864 & $230 \pm 15$ & 0.9915 \\
\hline 0.95 & $233 \pm 13$ & 0.9939 & $249 \pm 26$ & 0.9779 \\
\hline Mean & $251 \pm 21$ & 0.9836 & $241 \pm 27$ & 0.9681 \\
\hline
\end{tabular}

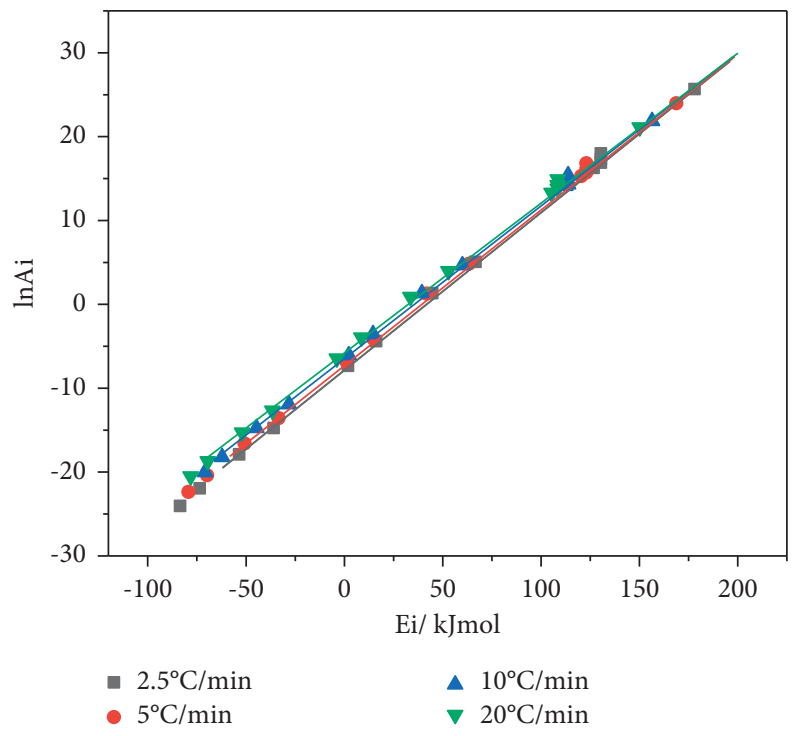

FIGURE 5: Observed compensation effect between the preexponential factor and apparent activation energy for thermal decomposition of the p-EGF:AA copolymer at different heating rates.

TABLE 4: Values of the compensation effect parameters of thermal decomposition of the p-EGF:AA copolymer at four different heating rates.

\begin{tabular}{lccr}
\hline$\beta\left({ }^{\circ} \mathrm{C} / \mathrm{min}\right)$ & $a^{*}$ & $b^{*}$ & $R^{2}$ \\
\hline 2.5 & 0.1979 & -5.8526 & 0.9989 \\
5 & 0.1956 & -5.3131 & 0.9994 \\
10 & 0.1922 & -4.6068 & 0.9994 \\
20 & 0.1887 & -3.8044 & 0.9994 \\
\hline
\end{tabular}




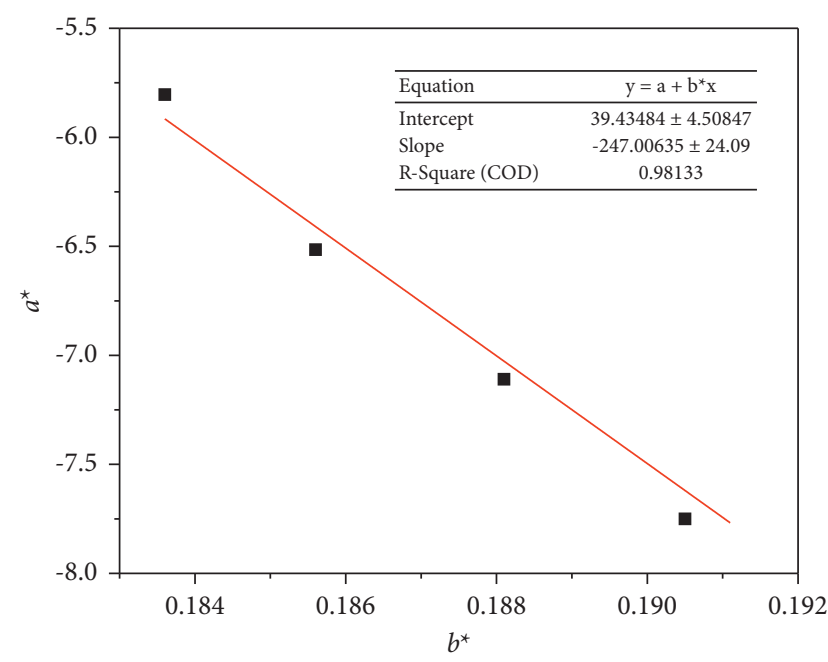

FIGURE 6: Graph of the supercorrelation ratio ( $a^{*}$ vs. $\left.b^{*}\right)$.

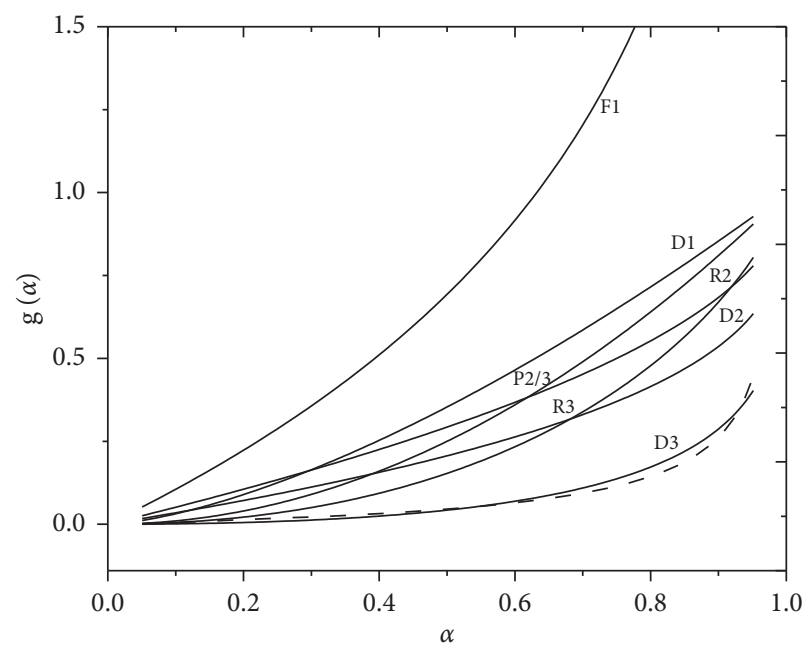

Figure 7: Theoretical curves $g(\alpha)$ as a function of $\alpha$ transformation for several kinetic models and the experimental graph for the kinetic model D3.

\section{Conclusion}

A more in-depth study of the thermal decomposition of the copolymer based on polyethylene glycol fumarate with acrylic acid was carried out in this work. Analysis of TG and DTG curves showed sufficient thermal stability of those copolymers in a nitrogen atmosphere. The decomposition took place in two stages. The temperature range of the main stage was about $260-460^{\circ} \mathrm{C}$.

The kinetic parameters of the decomposition reaction, which have good convergence, have been calculated using the Friedman and Kissinger-Akahira-Sunose isoconversion methods. It should be noted that the obtained data on the activation energy have close values at different heating rates. Using a combination of different methods, a kinetic triplet corresponding to $E a \approx 250 \mathrm{~kJ} / \mathrm{mol}, A \approx 8.66 \cdot 10^{16} \mathrm{~min}^{-1}$, and $\left.g(\alpha)=[1-(1-\alpha)]^{1 / 3}\right]^{2}$ was obtained. The dependences of $g(\alpha)$ on $\alpha$ using these parameters showed satisfactory agreement between the calculated and experimental curves. Thus, it can be said that the decomposition process of the p-EGF:AA copolymer is well described by the D3 model (three-dimensional diffusion).

\section{Data Availability}

The data used to support the findings of this study are available from the corresponding author upon request.

\section{Conflicts of Interest}

The authors declare that they have no conflicts of interest.

\section{Acknowledgments}

This research was funded by the Science Committee of the Ministry of Education and Science of the Republic of Kazakhstan (Grant No. BR10965249).

\section{References}

[1] S. Vyazovkin, A. K. Burnham, J. M. Criado, L. A. PerezMaqueda, C. Popescu, and N. Sbirrazzuolli, "ICTAC kinetic committee recommendation for performing kinetic computations on thermal analysis data," Thermochimica Acta, vol. 520, no. 1-2, pp. 1-19, 2011.

[2] T. Gikarakis, I. Pappas, P. Arvanitaki et al., "Thermal stability and kinetics of thermal decompositions of statistical copolymers of $\mathrm{N}$-vinylpyrrolidone and alkyl methacrylates synthesized via RAFT polymerizations," Journal of Chemistry, vol. 2021, Article ID 6633052, 12 pages, 2021.

[3] S. Vyazovkin, K. Chrissafis, M. L. Di Lorenzo et al., "ICTAC kinetics committee recommendations for collecting experimental thermal analysis data for kinetic computations," Thermochimica Acta, vol. 590, pp. 1-23, 2014.

[4] B. Wunderlich, Thermal Analysis of Polymeric Materials, Springer, Berlin, Germany, 2005.

[5] W. W. Wendlandt, Thermal Methods of Analysis, Wiley, Hoboken, NJ, USA, 1974.

[6] A. M. Elsharif, A. A. Al-Ghamdi, and A. S. Abdel-Naby, "Modification of poly (vinyl chloride) by N-phenyl itaconimides for the improvement of its thermal stability," Journal of Chemistry, vol. 2019, Article ID 4782745, 8 pages, 2019.

[7] H. V. Boenig, Unsaturated Polyesters: Structure and Properties, Elsevier, Amsterdam, Netherlands, 1964.

[8] M. Z. Burkeev, E. M. Tazhbaev, G. K. Kudaibergen et al., "Constants and parameters of radical copolymerization of poly (propylene glycol) fumarate with acrylic acid," Bulletin of the Karaganda University "Chemistry" Series, vol. 93, no. 1, pp. 25-31, 2019.

[9] M. Z. Burkeev, G. K. Kudaibergen, G. K. Burkeeva et al., "New polyampholyte polymers based on polypropylene glycol fumarate with acrylic acid and dimethylaminoethyl methacrylate," Russian Jornal of Applied Chemistry, vol. 91, no. 7, pp. 1145-1152, 2019.

[10] A. Z. Sarsenbekova, E. A. Zhortarova, M. Z. Burkeev, E. M. Tazhbaev, and A. T. Kazhmuratova, "Molecular masses and characteristics of polyethileneglycolmaleate, polypropyleneglycolmaleate, polyethileneglycolphthalate, polypropyleneglycolphthalate," Bulletin of University of Karaganda. Series Chemistry, vol. 74, no. 2, pp. 15-20, 2014. 
[11] S. V. Vinogradova, V. V. Korshak, V. I. Gribkova, and V. G. Danilov, "Thermal stability of unsaturated polyesters and copolymers based on them," Polymer Science U.S.S.R.vol. 10, no. 7, pp. 1757-1764, 1968.

[12] K. W. Ewa, "Kinetics of thermal decomposition of unsaturated polyester resins with reduced flammability," Journal of Applied Polymer Science, vol. 88, no. 13, pp. 2851-2857, 2003.

[13] M. Z. Burkeev, A. Z. Sarsenbekova, Y. M. Tazhbayev, and I. V. Figurinene, "Thermal destruction of polypropylene glycolmaleinate copolymers with acrylic acid," Russian Journal of Physical Chemistry A, vol. 89, no. 12, pp. 2183-2189, 1968.

[14] A. Z. Sarsenbekova, G. K. Kudaibergen, M. Z. Burkeev, and G. K. Burkeeva, "Comparative analysis of the thermal decomposition kinetics of polyethylene glycol fumarate-acrylic acid copolymers," Russian Journal of Physical Chemistry A, vol. 93, no. 7, pp. 1252-1257, 2019.

[15] M. Z. Burkeev, A. N. Bolatbay, A. Z. Sarsenbekova, S. Z. Davrenbekov, and E. Nasikhatuly, "Integral ways of calculating the destruction of copolymers of, 2021 of polyethylene glycol fumarate with acrylic acid," Russian Journal of Physical Chemistry A, vol. 95, no. 10, pp. 1496-1500, 2021.

[16] N. V. Muravyev, A. N. Pivkina, and N. Koga, "Critical appraisal of kinetic calculation methods applied to overlapping multistep reactions," Molecules, vol. 24, no. 12, p. 2298, 2019.

[17] M. Z. Burkeyev, G. K. Kudaibergen, Y. M. Tazhbayev et al., "The number average and mass average molar masses of polyethylene (propylene) glycol fumarates," Bulletin of University of Karaganda Series Chemistry, vol. 90, no. 2, pp. 17-22, 2018.

[18] G. K. Kudaibergen, G. K. Burkeyeva, Y. M. Tazhbayev, M. Z. Burkeyev, A. V. Omasheva, and N. A. Yesentayeva, "Constants and parameters of radical copolymerization of poly (ethylene glycol fumarate) with acrylic acid," Chemical Journal of Kazakhstan, vol. 61, no. 1, pp. 216-222, 2018.

[19] H. E. Kissinger, "Reaction kinetics in differential thermal analysis," Analytical Chemistry, vol. 29, no. 11, pp. 1702-1706, 1957.

[20] T. Akahira and T. Sunose, "Method of determining activation deterioration constant of electrical insulating materials," Research Report, pp. 22-31, Chiba Institute of Technology, Chiba, Japan, 1971.

[21] H. L. Friedman, "Kinetics of thermal degradation of charforming plastics from thermogravimetry. Application to a phenolic plastic," Journal of Polymer Science, vol. 6, no. 1, pp. 183-185, 1964.

[22] B. B. Achar, G. W. Brindley, and J. H. Sharp, "Kinetics and mechanism of dehydroxylation processes, III. Applications and limitations of dynamic methods," in Proceedings of the International Clay Conference, L. Heller and A. Weiss, Eds., pp. 67-73, Israel Program for Scientific Translations, Jerusalem, Israel, 1966.

[23] A. I. Lesnikovich and S. V. Levchik, "A method of finding invariant values of kinetic parameters," Journal of Thermal Analysis, vol. 27, no. 1, pp. 89-93, 1983.

[24] N. Sbirrazzuolli, "Determination of pre-exponential factors and of the mathematical functions $f(\alpha)$ or $G(\alpha)$ that describe the reaction mechanism in a model-free way," Thermochimica Acta, vol. 564, pp. 59-69, 2013. 